\title{
Early-type galaxies with core collapse supernovae
}

\author{
A. A. Hakobyan ${ }^{1}$, A. R. Petrosian ${ }^{1}$, B. McLean ${ }^{2}$, D. Kunth ${ }^{3}$, R. J. Allen ${ }^{2}$, M. Turatto ${ }^{4}$, and R. Barbon ${ }^{5}$ \\ 1 Byurakan Astrophysical Observatory and Isaac Newton Institute of Chile, Armenian Branch, Byurakan 378433, Armenia \\ e-mail: hakartur@rambler.ru \\ 2 Space Telescope Science Institute, 3700 San Martin Drive, Baltimore, MD 21218, USA \\ 3 Institut d'Astrophysique de Paris (UMR 7095: CNRS \& Université Pierre et Marie Curie), 98bis Bd Arago, 75014 Paris, France \\ 4 INAF, Osservatorio Astrofisico di Catania via Santa Sofia 78, 95123 Catania, Italy \\ 5 Dipartimento di Astronomia, Università di Padova, vicolo dell'Osservatorio 2, 35122 Padova, Italy
}

Received 20 March 2008 / Accepted 16 June 2008

\section{ABSTRACT}

\begin{abstract}
Aims. It is widely accepted that the progenitors of core collapse SNe are young massive stars and therefore their host galaxies are mostly spiral or irregular galaxies dominated by a young stellar population. Surprisingly, among morphologically classified hosts of core collapse SNe, we find 22 cases where the host has been classified as an Elliptical or S0 galaxy.

Methods. To clarify this apparent contradiction, we carry out a detailed morphological study and an extensive literature search for additional information on the sample objects.

Results. Our results are as follows:

1. Of 22 "early type" objects, 17 are in fact misclassified spiral galaxies, one is a misclassified irregular, and one is a misclassified ring galaxy.

2. Of the 3 objects maintaining the early type classification, one (NGC 2768) is a suspected merger remnant, another (NGC 4589) is definitely a merger, and the third (NGC 2274) is in close interaction. The presence of some amount of young stellar population in these galaxies is therefore not unexpected.

Conclusions. These results confirm the presence of a limited, but significant, number of core collapse SNe in galaxies generally classified as early-type. In all cases, anyway, there are independent indicators of the presence in host galaxies of recent star formation due to merging or gravitational interaction.
\end{abstract}

Key words. galaxies: general - supernovae: general

\section{Introduction}

As their cosmological importance as distance indicators continues to grow, a detailed understanding of the origin of supernovae (hereafter $\mathrm{SNe}$ ) is becoming one of the more perplexing problems in modern astronomy. Especially in the last two decades, improvements in observational capabilities and the broader spectral range of $\mathrm{SNe}$ studies have resulted in an unexpected diversity of the observed properties of SNe leading to the introduction of new types and subtypes. These are likely to be related to different physical parameters of the stellar progenitors of $\mathrm{SNe}$ (possibly leading to observable differences in the details of their explosion mechanisms), and to differences in the properties of their immediate environment (e.g., Turatto et al. 2007). Current limits on our understanding of these issues make it difficult to come to firm conclusions on the role of $\mathrm{SNe}$ in several fields like the chemical evolution of galaxies, the production of the stellar remnants and the origin of cosmic rays. Perhaps most vexing are the doubts that remain on the reliability with which SNe can be used as distance indicators up to cosmological distances.

It is widely accepted that $\mathrm{SNe}$ result from two major explosion mechanisms: core collapse in massive stars $\left(M \geq 8 M_{\odot}\right)$ and thermonuclear runaway of in a WD approaching the Chandrasekar limit by accretion from a companion. The focus of this paper is on $\mathrm{SNe}$ formed via core collapse (hereafter CCSNe). Different configurations of the progenitor at the moment of explosion, different energetics associated with the event, and possible interaction of the ejecta with circumstellar material produce a large variety of characteristics, presently classified as SNe of types Ib and Ic and several sub-types of type II. Spectacular evidence for the core collapse nature of SNe II comes from the detection of neutrinos of SN1987A in LMC but indication that $\mathrm{CCSNe}$ have massive progenitors also comes from about a dozen cases of direct identification of core collapse SN progenitors (e.g., van Dyk 2005; Hendry et al. 2006), from theoretical modeling (e.g., Woosley \& Weaver 1995; Heger et al. 2003), and from studies of the environment of these SNe. Their association with spiral arms in galaxies (e.g., Maza \& van den Bergh 1976; Bartunov et al. 1994), with massive starforming regions (e.g., van Dyk 1992), and with local young stellar population (e.g., van Dyk et al. 1999) is well known. This is consistent with the dependence of the rate of explosions with the morphological types of the host galaxies. Many studies have concluded that types II and $\mathrm{Ib} / \mathrm{c}$ SNe occur only in spiral and irregular galaxies, and mostly in those of the latest types (e.g., Cappellaro et al. 1999). In other words different studies show that the progenitor stars of CCSNe are young massive stars born in recent episodes of star formation in their host galaxies (e.g., Hamuy 2003), with possible differences in their nature (e.g., van Dyk et al. 2003). Conversely, the apparent certainty of the association between CCSNe and young massive stars has led to use CCSNe as tracers of recent star formation in galaxies (e.g., Navasardyan et al. 2001; Petrosian et al. 2005).

It is therefore puzzling that several authors have reported the presence of CCSNe in E or S0 galaxies. In three articles, 
van den Bergh et al. (2002, 2003, 2005, hereafter vdBLF) discussed the morphologies of host galaxies in the Lick Observatory Supernova Search (LOSS; e.g., Filippenko et al. 2001) and report 8 cases of E and S0 galaxies harboring CCSNe. In a new examination of the objects listed in the latest version (October 30, 2007) of the Asiago Supernova catalog (ASC; e.g., Barbon et al. 1999) we discovered 14 more cases of CCSNe host galaxies classified as type E or S0. If demonstrated, the presence of CCSNe in early type galaxies should be associated with the population of the prompt SN Ia advocated by Mannucci et al. (2006) to explain the excess of SN Ia events observed in radioloud ellipticals. This excess is attributed to recent episodes of massive star formations which are expected to produce CCSNe although in still debated number (Greggio et al. 2008).

It is known that type Ib/c SNe can be misclassified as SN Ia because of the similarity of their spectra at some epoch. A recent example is SN2004aw (Taubenberger et al. 2006). It is not excluded therefore that some less extensively studied SN Ia reported in early type galaxies, might indeed be misclassified $\mathrm{SN} \mathrm{Ib/c.} \mathrm{However,} \mathrm{since} \mathrm{to} \mathrm{our} \mathrm{knowledge} \mathrm{a} \mathrm{statistics} \mathrm{of} \mathrm{these}$ cases is not available, we neglect such possible effect in what follows. In this paper we study in detail all 22 cases with the aim of understanding the reliability of the host galaxy classification. The sample is presented in more detail in Sect. 2. The results of our study of individual host galaxies are presented in Sect. 3, and in Sect. 4 we discuss and summarize our results. Throughout this article we have assumed a value of $H_{0}=75 \mathrm{~km} \mathrm{~s}^{-1} \mathrm{Mpc}^{-1}$ for the Hubble constant.

\section{The sample}

The three papers of vdBLF discussed the morphological classification of more than 600 host galaxies of SNe. This classification was done on the DDO system using images from the $0.75 \mathrm{~cm}$ Katzman Automatic Imaging Telescope during the course of the Lick Observatory Supernova Search as well as from the POSS-I, POSS-II, or SERC-J and SERC-R blue and red exposures. The Asiago Supernova Catalog ${ }^{1}$ referenced above has presently (October 30, 2007) accumulated data for about $4300 \mathrm{SNe}$ and their host galaxies and it includes (among other parameters) the morphological classes of the hosts. These come from various sources, mostly from RC3 (de Vaucouleurs et al. 1991) and LEDA (70\% and $20 \%$ respectively). It is interesting to note that the classifications are not always in agreement: of the 8 cases reported by vdBLF four have been classified as spirals in the ASC; conversely, three galaxies of the 14 from the ASC are classified as spirals (or possible spirals) by vdBLF. We have therefore considered the full list of 22 cases for our study for which an Elliptical or S0 type parent galaxy is suspected. It is worth mentioning that we have not considered S0/a type galaxies for this study. There are two crucial reasons for this decision: firstly S0/a type galaxies are well known to have an increasingly large young stellar population (e.g., Noordermeer et al. 2005) and can harbor CCSN progenitors; secondly the distinction between $\mathrm{S} 0$ and S0/a's is subtle and give rise to possible misclassification (e.g., Naim et al. 1995). It is interesting to note that vdBLF did not report any CCSN in S0/a type galaxy but in ASC there are 15 such galaxies with 15 CCSNe. Table 1 presents the list of 22 cases with CCSNe identified in E or S0 type galaxies. The first column gives the name of the host galaxy sorted according to RA, the second and third columns list the supernovae and their classifications from ASC. In three cases two SNe events

${ }^{1}$ http://web.oapd.inaf.it/supern/cat were discovered in the same galaxy and it is remarkable that in one (NGC 2836) both events where core collapse explosions. Columns 4 and 5 give the morphological types of the hosts according to vdBLF and to the ASC where ":" denote uncertain classifications and "?" very uncertain ones. Our remarks and the new galaxy types are given in Col. 6 .

\section{The morphological classification}

We have taken a fresh approach to classify the host galaxies in Table 1 as in Petrosian et al. (2007, 2008). We extracted their images from the POSS-II or SERC blue and red original plates digitally archived at STScI. Since the original plate material was intended for the measurement of stellar objects between 12 and $22 \mathrm{mag}$ in blue and between 12 and $21 \mathrm{mag}$ in red, it is suitable for a morphological study of the sample galaxies, which are mostly bright objects. For some very bright galaxies (brighter than $14 \mathrm{mag}$ ) and high surface brightness objects, the central parts of their images may be over-exposed, but for most galaxies the central structure is well displayed. This plate material is therefore especially useful for examining the structural features of the galaxies both in their inner and their outer regions. A morphological classification of each galaxy was done first on red images and later checked on the blue ones. We have carried out the classification by using gray-scale displays of the digitized images and by inspecting isophotal maps. The latter ones are especially useful in representing the large dynamical range in the images. The appearance of both the central and the outer regions of the galaxies has been taken into account in arriving at a classification. In Fig. 1 we have collected gray-scale and contour diagram representations of the $F$ band images of our sample galaxies. These images are shown in figures of two images each, comprising 22 sample galaxies. The contour levels are in arbitrary units of photographic density. The lowest contour level was chosen at about the $3 \sigma$ level of the local background. The contour interval is constant, different in each galaxy, but usually between $10-30 \%$ of the local background and chosen in order to best illustrate both the inner and the outer structure of the galaxy. In the same fashion, the field size (and thus magnification) was selected for each system to clearly illustrate its morphological structure. In all images, the precise positions of the relevant $\mathrm{SNe}$ are also shown. These positions have been calculated according to the offsets given in ASC and verified on the available finding charts of the $\mathrm{SNe}$ when available in the WEB. For each galaxy of the sample we also conducted an extensive literature search in order to find other information on its morphology as well as any additional evidence for the presence of ongoing star formation. In the following we present the individual discussion on the morphology of each host galaxy of our sample.

IC 1529. Although listed as a So galaxy in the ASC, in Huchra et al. (1993), and in da Costa et al. (1998), NED classifies this galaxy as $\left(\mathrm{R}^{\prime}\right) \mathrm{SA}(\mathrm{r}) 0^{\wedge} 0^{\wedge}$ pec:. This object is a UV-bright galaxy ( $U-B=-0.5$ ), with prominent $\mathrm{H} \alpha$ and [NII] emission line spectra and it is included in the second list of the Montreal Blue Galaxy Survey (object MBG0002-1146; Coziol et al. 1994). According to Meyer et al. (2004) this galaxy shows strong $21 \mathrm{~cm} \mathrm{HI}$ emission, and Mauch \& Sadler (2007) list it as a $1.4 \mathrm{GHz}$ radio continuum source. The presence of such tracers is already indicative of enhanced star formation in this galaxy (e.g., Kennicutt 1989). Mauch \& Sadler (2007) classified the optical spectra of this galaxy as narrow LINER-like emission-line spectra. According to our image study, we classify this galaxy as Sbc type with low surface brightness spiral arms and with a high surface brightness, disturbed central bulge structure. SN2006du 
Table 1. The list of early type galaxies with core collapse supernovae.

\begin{tabular}{|c|c|c|c|c|c|}
\hline \multirow{2}{*}{$\begin{array}{c}\text { Galaxy } \\
\text { (1) }\end{array}$} & \multirow{2}{*}{$\begin{array}{l}\text { SN } \\
\text { (2) }\end{array}$} & \multirow{2}{*}{$\begin{array}{l}\text { SN type } \\
\text { (3) }\end{array}$} & \multicolumn{2}{|c|}{ Morphology } & \multirow{2}{*}{$\begin{array}{c}\text { New galaxy types and remarks } \\
\text { (6) }\end{array}$} \\
\hline & & & $\begin{array}{l}\text { vdBLF } \\
(4)\end{array}$ & $\begin{array}{l}\text { ASC } \\
(5)\end{array}$ & \\
\hline IC 1529 & 2006du & II & & S0 & $\begin{array}{l}\text { Sbc type galaxy with LINER type AGN } \\
\text { HI, } 1.4 \mathrm{GHz} \text { radio source }\end{array}$ \\
\hline NGC 774 & 2006ee & II & & So & So. Embryonic spiral arm exist. Residual star formation \\
\hline NGC 838 & $2005 \mathrm{H}$ & II & & S0: pec & Mrk1022. Im type galaxy. HI, $1.4 \mathrm{GHz}$ radio source \\
\hline MCG-01-07-35 & $2002 \mathrm{aq}$ & II & SBab & SB0 pec & Barred-Ring galaxy. HI source \\
\hline PGC 10652 & $2006 a b$ & Ic & & E? & Sb type galaxy. HI source \\
\hline IC 1861 & $1999 \mathrm{eg}$ & II & & So & Sbc type galaxy. HI source \\
\hline NGC 1260 & 2006gy & IIn? & & S0: & Sa. Extended $\mathrm{H} \alpha$ emission also at the $\mathrm{SNe}$ position \\
\hline IC 340 & $2002 \mathrm{jj}$ & Ic & S pec? & S0: & Sc type galaxy. HI source \\
\hline UGC 2836 & $\begin{array}{l}2001 \mathrm{I} \\
2003 \mathrm{ih}\end{array}$ & $\begin{array}{l}\mathrm{IIn} \\
\mathrm{Ib} / \mathrm{c}\end{array}$ & E1 & So & $\begin{array}{l}\text { Mrk1405. Sa type galaxy. } \mathrm{HI}, \mathrm{H}_{2}, \mathrm{CO} \text { and } \\
1.4 \mathrm{GHz} \text { radio source }\end{array}$ \\
\hline UGC 3432 & $\begin{array}{l}2003 \mathrm{~kb} \\
1996 \mathrm{bv}\end{array}$ & $\begin{array}{l}\text { Ic: } \\
\text { Ia }\end{array}$ & $\mathrm{S} 0 / \mathrm{Sb}$ & Scd: & Sc type galaxy. HI source \\
\hline NGC 2274 & 2005md & II: & & $\mathrm{E}$ & E type galaxy in interaction. HI source \\
\hline NGC 2768 & 2000ds & $\mathrm{Ib}$ & $\mathrm{E} 3 / \mathrm{Sa}$ & E6 & $\begin{array}{l}\text { S0 type galaxy with LINER type AGN. HI, CO and } \\
1.4 \mathrm{GHz} \text { radio source. A possible merger remnant }\end{array}$ \\
\hline IC 2461 & $2002 b x$ & II & So & $\mathrm{Sb}$ & $\begin{array}{l}\text { Sbc type galaxy. } \mathrm{HI} \text { and } 1.4 \mathrm{GHz} \text { radio source } \\
\text { with emission line spectrum }\end{array}$ \\
\hline VIIIZw140 & $2004 X$ & II & E3 & & Spiral galaxy? HI and $1.4 \mathrm{GHz}$ radio source \\
\hline NGC 3720 & 2002at & II: & E1 & Sa: & $\begin{array}{l}\text { Sa type galaxy. } \mathrm{HI} \text { and } 1.4 \mathrm{GHz} \text { radio source } \\
\text { with emission line spectrum }\end{array}$ \\
\hline IC 3203 & $2003 \mathrm{ac}$ & IIb: & So & $\mathrm{Sb}$ & Sb type galaxy. HI source \\
\hline ESO 506-G11 & 2005an & II: & & So & Sb type galaxy \\
\hline NGC 4589 & $2005 \mathrm{cz}$ & $\mathrm{Ib}$ & & E2 & $\begin{array}{l}\text { Merger with LINER type AGN. HI and } \\
1.4 \mathrm{GHz} \text { radio source }\end{array}$ \\
\hline ESO 576-G40 & $\begin{array}{l}2003 \mathrm{am} \\
1997 \mathrm{br}\end{array}$ & $\begin{array}{l}\text { II } \\
\text { Ia pec }\end{array}$ & So t & & $\begin{array}{l}\text { SBc type galaxy. HI source with } \\
\text { emission line spectrum }\end{array}$ \\
\hline MAPS-NGP O_442_2131626 & 2004V & II & E0 & & Sa type galaxy \\
\hline UGC 12044 & $2002 \mathrm{hz}$ & $\mathrm{Ib} / \mathrm{c}$ & $\mathrm{Sb} / \mathrm{S} 0$ & Sab & Sab type galaxy. HI source \\
\hline MCG-02-57-22 & 2001hh & II & $\mathrm{Sa}$ & SB0: & Sa type galaxy \\
\hline
\end{tabular}

is a classical SN II discovered close to maximum (Green 2006) and located on an outer spiral arm.

NGC 774. According to the ASC, this is a S0 galaxy, and the same morphological type is reported in NED and also by Huchra et al. (1999). Caldwell et al. (2003) studied the stellar population of this galaxy and concluded that there is no significant young stellar population. This galaxy has been observed by the Sloan Digital Sky Survey (SDSS; e.g., AdelmanMcCarthy et al. 2007), and a direct image and spectra are presented in latest SDSS ${ }^{2}$ Data Release 6 (DR6) (object SDSS J015934.72+140029.5). The nuclear region of this galaxy shows faint [NII]6584 and a trace of $\mathrm{H} \alpha$ lines in emission. The SDSS images and our own are in agreement with previous determinations. According to its outer isophotal structure and its radial distribution of surface brightness, this is a S0 type object. However, the distribution of surface brightness shows some small degree of asymmetry in the region to the south-southwest of the nucleus. Here, we suspect the presence of an embryonic spiral arm. SN2006ee is a type II SN (Puckett et al. 2006) with plateau ${ }^{3}$, exploded very close to this region.

NGC 838. According to ASC, NGC 838 is a S0pec type galaxy and the same morphological type $\left(\mathrm{SA}(\mathrm{rs}) 0^{\wedge} 0 \mathrm{pec}\right)$ is reported in NED. The galaxy being member of Arp318 peculiar system is also member of Hickson compact group of galaxies (HCG16C; Hickson et al. 1989) and classified as Im type object. According to the de Carvalho \& Coziol (1999) it is

\footnotetext{
2 http://www.sdss.org/dr6/

${ }^{3}$ http://csp1.1co.cl/ cspuser1/PUB/PROJ/LC/c3_op/ SNQ6ee.html
}

Im type galaxy with double nuclei structure and with narrow emission line spectra. The double nuclei structure and irregular morphology is well seen also on the SDSS DR6 image (SDSS J020938.55-100847.5). As an UV excess galaxy it is also known as Markarian 1022 (also is KUG0207-103) which Petrosian et al. (2007) classified as Im type. This object has blue colour $(U-B=-0.06 ; B-V=0.58)$ and shows $1.4 \mathrm{GHz}$ radio continuum emission (Condon et al. 1998). From the analysis of Fig. 1 we confirm double nuclei irregular morphological structure of this galaxy. SN2005H was a normal type II discovered short after the burst (Pastorello et al. 2005), positioned outside the main body of this galaxy, in the low surface brightness diffuse bridge connecting NGC 838 with NGC 835 (Mrk1021 = HCG16A; Sab (Petrosian et al. 2007) galaxy). In the northern edge of this bridge, closer to NGC 835, an isolated HII region was discovered (Ryan-Weber et al. 2004). In all its length the bridge radiates in HI (Verdes-Montenegro et al. 2001).

MCG-01-07-035. NED assigns SB(r)0+ pec type to this galaxy and LEDA classifies it as SB0/a with outer ring (R) structure. The ASC reports it as a S0pec galaxy but van den Bergh et al. (2002) have classified it as SBab. According to Theureau et al. (2007) this galaxy is a $21 \mathrm{~cm}$ HI source. In Fig. 1 this galaxy shows an obvious bar and disturbed outer ring structure. SN2002aq, a normal SN II (Filippenko \& Barth 2002), was located in the inner edge of the outer ring.

PGC 10652. According to ASC PGC10652 is an elliptical (E?), to Hau et al. (1995) it is E/S0 while to NED it is Sb. Because of the strong HI emission Huchtmeier \& Richter (1989) included this object in their catalogue. Our morphological study confirms the double spiral arm structure of this galaxy and the 

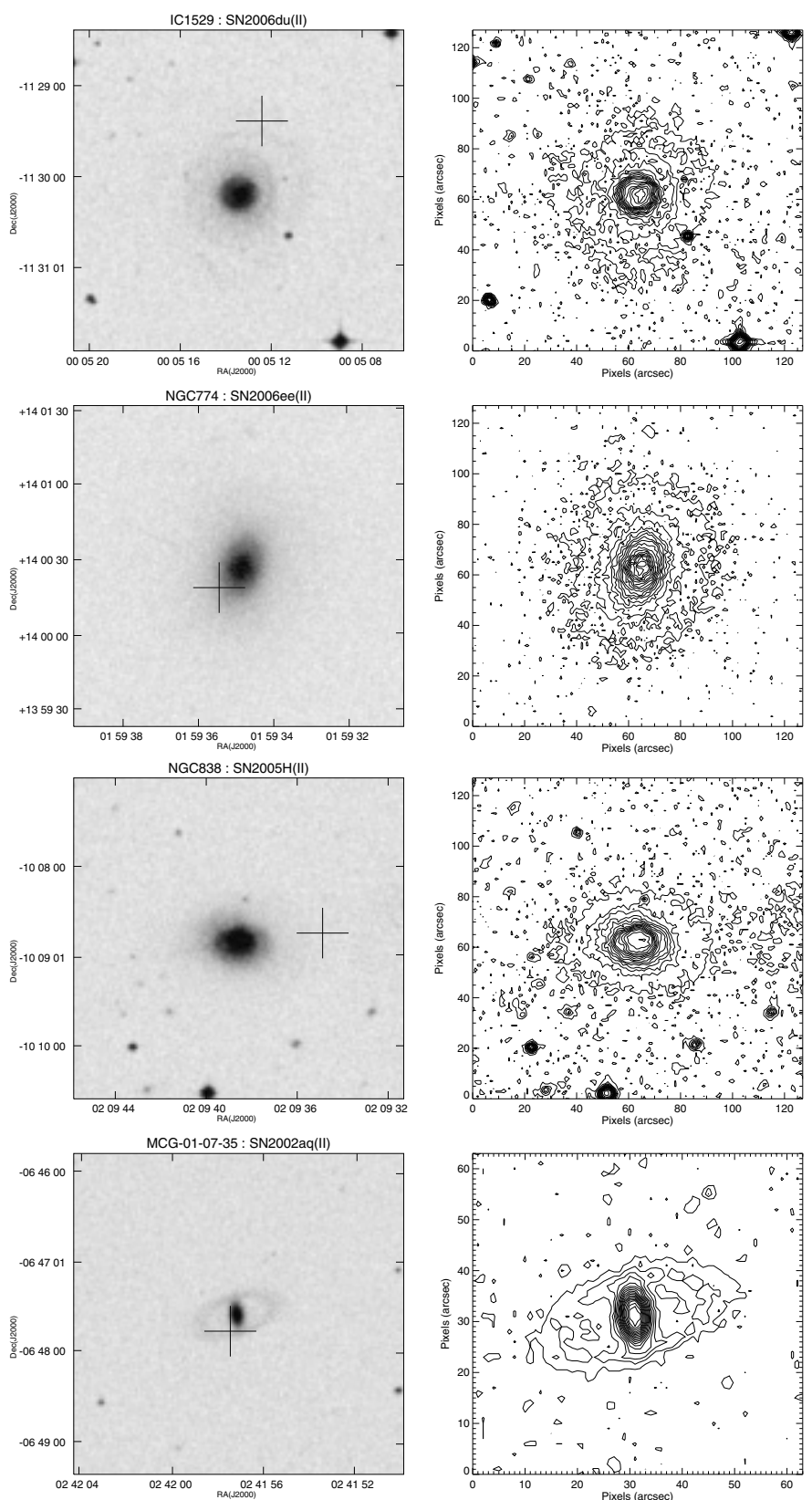

Fig. 1. a) Gray-scale and isophotal maps of early type galaxies with core collapse supernovae. The positions of SNe are marked by "+" cross sign.

Sb classification. SN2006ab, a type Ic SNe (Wong et al. 2006), was located close to the inner edge of the northern spiral arm of the galaxy.

IC 1861. This object is also known as the isolated galaxy KIG120 (Karachentseva 1973). According to ASC it is a S0 galaxy in agreement with NED (SA0^0^) and Huchra et al. (1999) $(T=-2$, L- or S0-). Higher resolution imaging of KIG120 led to the Sbc type classification (Sulentic et al. 2006) which better agrees with its strong HI emission (Huchtmeier \& Richter 1989). According to our morphological study the low surface brightness spiral structure of this galaxy is obvious and confirms the Sbc classification. SN1999eg was a normal SN II (Jha et al. 1999) with plateau light curve (Hamuy 2001) positioned close to the inner edge of the spiral arm of the galaxy.

NGC 1260. This galaxy with radial velocity of $5753 \mathrm{~km} \mathrm{~s}^{-1}$ belongs to the Perseus cluster (A426) and forms an interacting
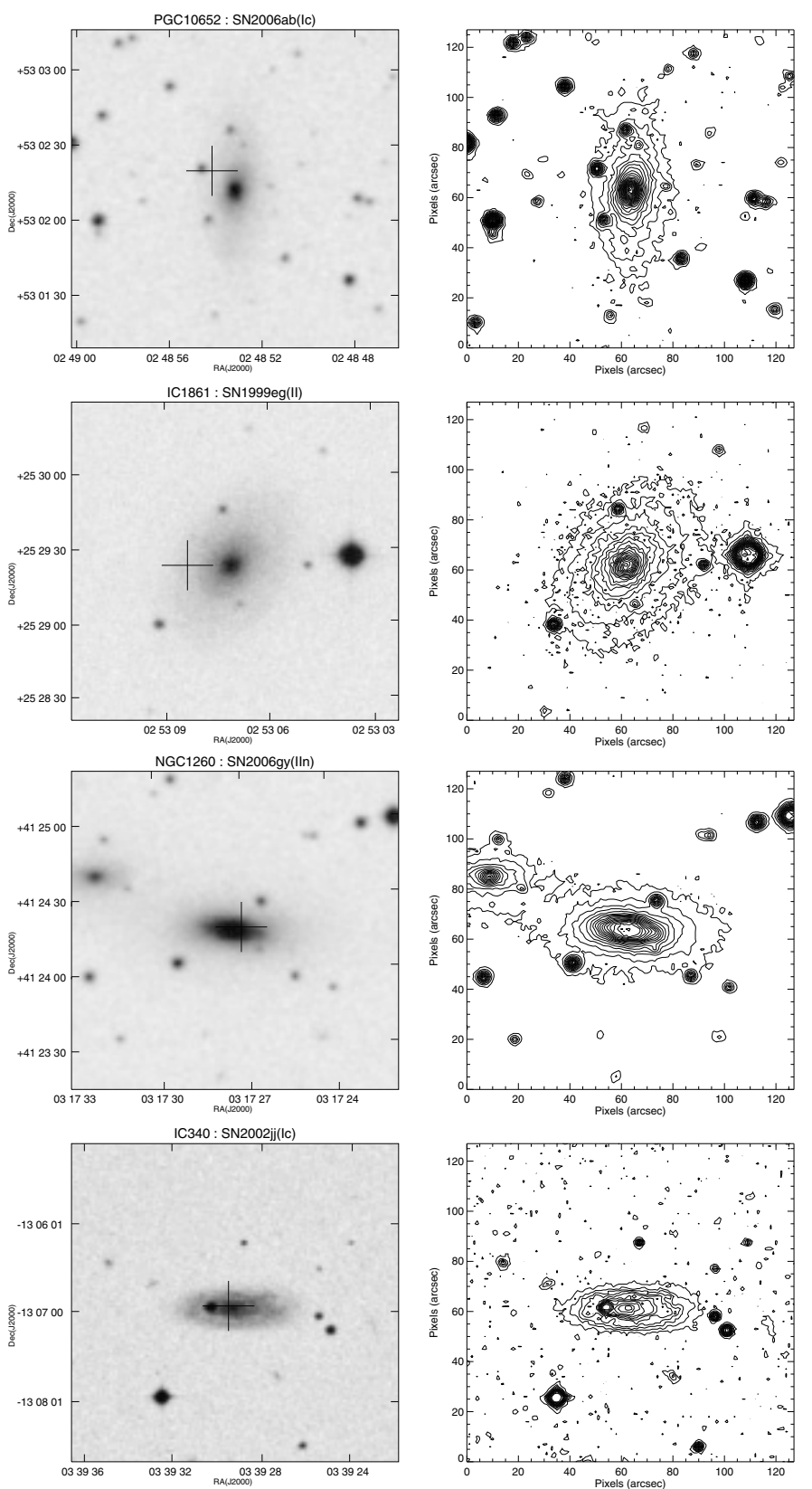

Fig. 1. b) continued.

pair with the close neighbor PGC $12230\left(V_{\mathrm{r}}=5957 \mathrm{~km} \mathrm{~s}^{-1}\right)$. ASC reports for this galaxy the S0: classification compatible with that reported by NED (S0/a:). Early type morphology $\mathrm{SB} / \mathrm{SB} 0(\mathrm{~T}=-1, \mathrm{~L}+$ or $\mathrm{S} 0+)$ is reported also Huchra et al. (1999) while according to Andreon et al. (1997) the morphological structure of this galaxy is similar to SA0 type objects with high amount of dust content in its inner part. An higher resolution and sensitivity morphological study of this galaxy by Meusinger et al. (2000) has shown weak indications of an outer spiral and bar structure. The presence of a short bar and a weak spiral arm structure in this galaxy is confirmed with our imagery study. We prefer to classify this galaxy as Sa type spiral. The discovery of SN2006gy very close to the nuclear region prompted very high resolution imaging of the parent galaxy in the optical (Fig. 3 of Ofek et al. 2007) and NIR (Smith et al. 2007). The resulting inner structure of the galaxy is that of an early type galaxy with Sersic profile index 3.7 (Ofek et al. 2007). Nevertheless, the presence of a consistent dust lane and extended 

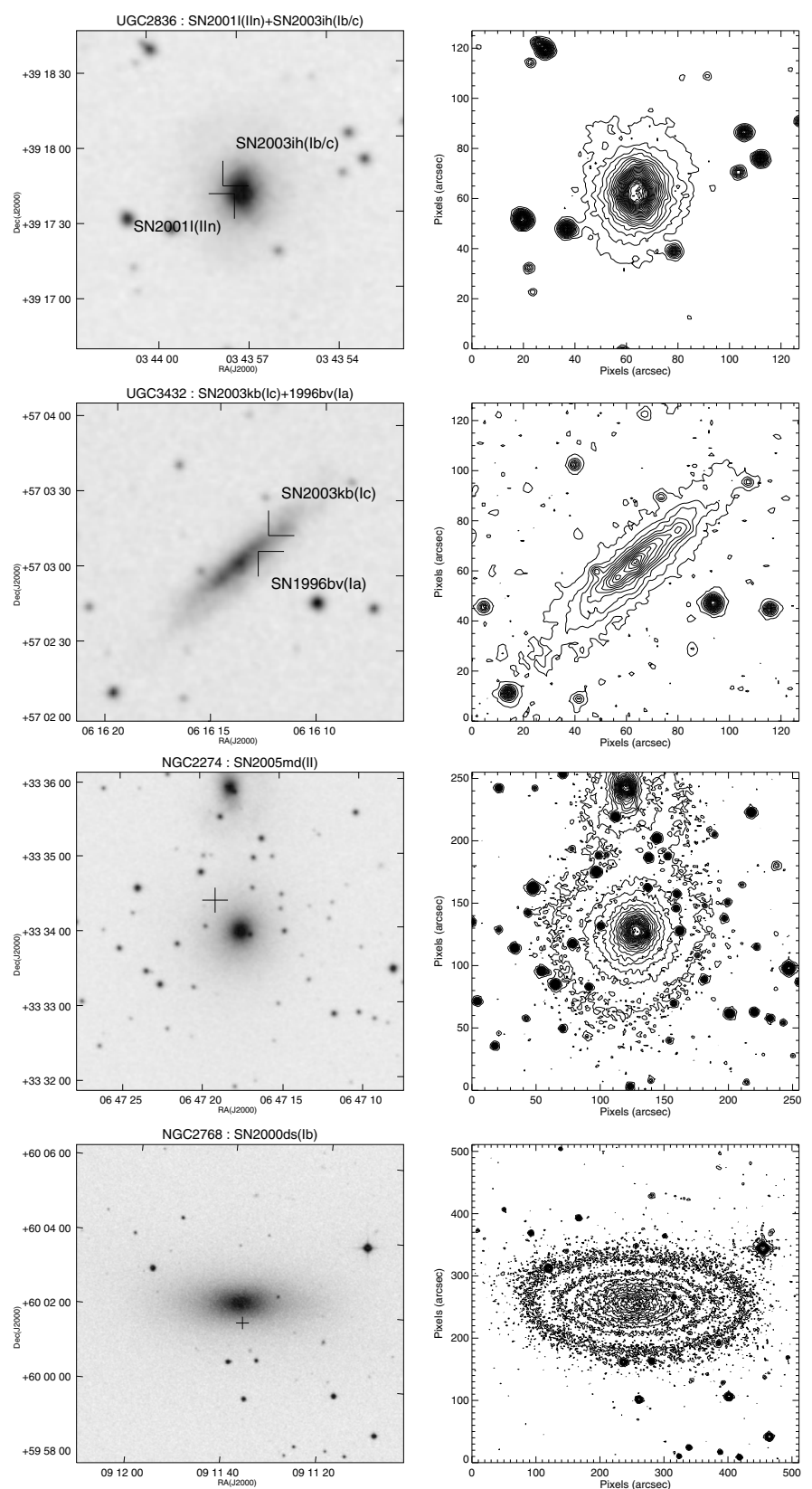

Fig. 1. c) continued.

$\mathrm{H} \alpha$ emission (Smith et al. 2007) are a clear evidence of ongoing star formation. SN2006gy is unique in many respects and it is the brightest SN ever observed (Ofek et al. 2007; Smith et al. 2007; Agnoletto et al. 2008, in preparation). Although the mechanism responsible for such explosion is still debated (Ofek et al. 2007; Smith et al. 2007; Woosley et al. 2007; Agnoletto et al. 2008) there is a general consensus that the progenitor was very massive $\left(M>40 M_{\odot}\right)$.

IC 340. According to the ASC this is a S0pec galaxy. van den Bergh et al. (2003) suspected its spiral nature and classified it as a Spec? object. NED reports type $\mathrm{S}^{\wedge} 0^{\wedge}$ while da Costa et al. (1998) classified it as type Sc. According to Theureau et al. (2007), this object is a strong HI source. Our inspection of the images of the galaxy confirms the spiral nature of the classification and we assign a type Sc. The type Ic SN2002jj (Foley \& Filippenko 2002) is positioned very close to the nuclear region.

UGC 2836. This object is Markarian 1405, which Petrosian et al. (2007) classified as Sa. The ASC considers it as a S0 and
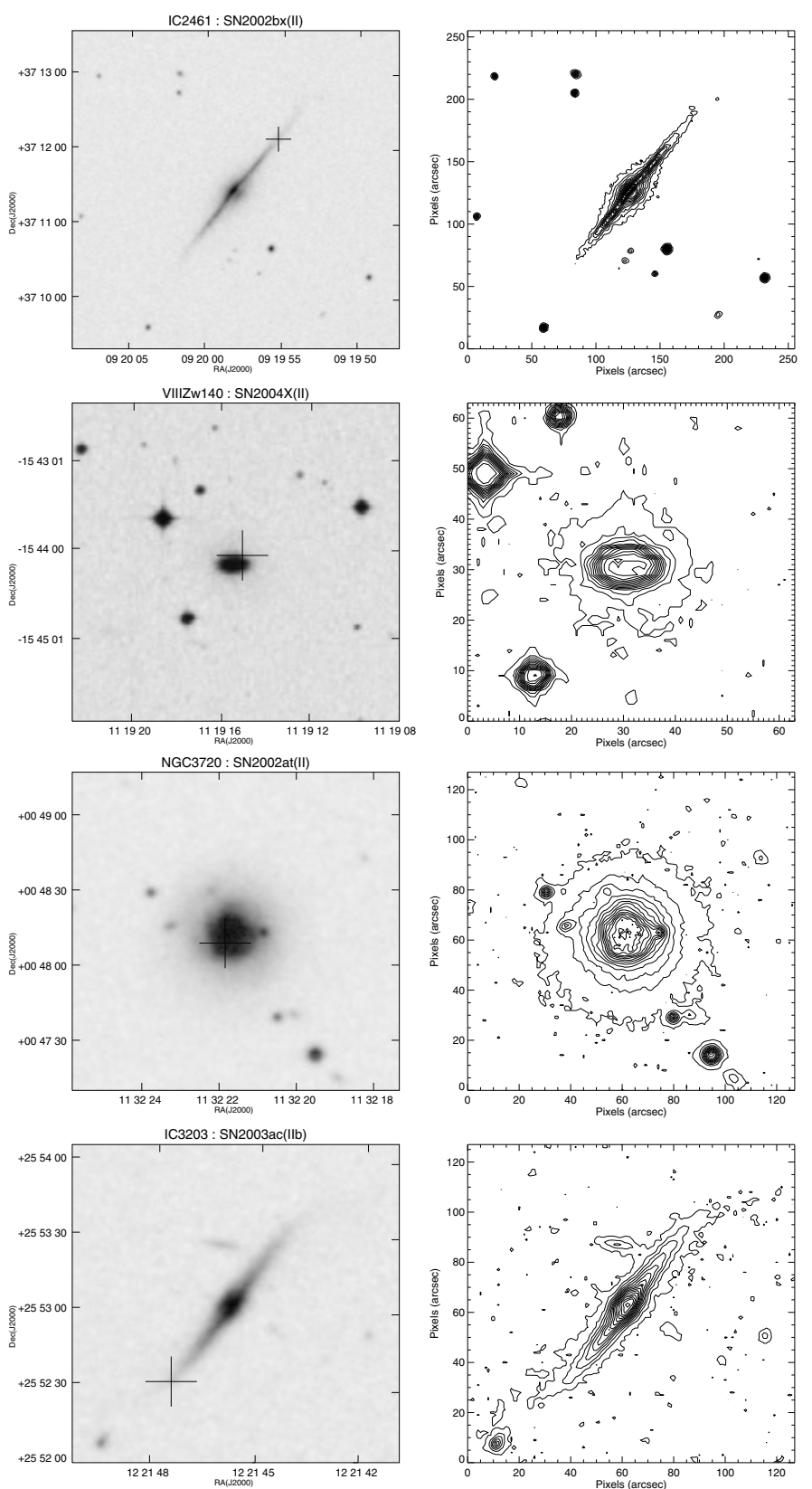

Fig. 1. d) continued.

NED as a S0-. van den Bergh et al. (2002) assigned type E1 but later decided that the classification $\mathrm{Sa}$ : was more appropriate (van den Bergh et al. 2003). According to Kandalyan (2003) this galaxy is a strong source of neutral $\mathrm{HI}$ and molecular $\mathrm{H}_{2}$, and $\mathrm{CO}$ emissions and a source of $1.4 \mathrm{GHz}$ continuum radio emission (Condon et al. 1998). Our morphological study confirms the Sa spiral structure of this object. Two core collapse SNe, the type IIn SN2001I (Filippenko \& Chornock 2001) and the type Ib/c SN2003ih (Filippenko \& Chornock 2003c), have been discovered in this galaxy. SN2001I lies closer to the nuclear region of the galaxy than SN2003ih. SN2003ih is located in the spiral pattern of the galaxy. Identification of the background structure of the region of the host galaxy associated with the SN2001I is problematic.

UGC 3432. The Scd: classification reported by ASC from RC3 and by NED (Gallagher et al. 2005) and the S0/Sb classification by van den Bergh et al. (2005) are contradictory enough to warrant a re-examination of the morphology of this galaxy. 

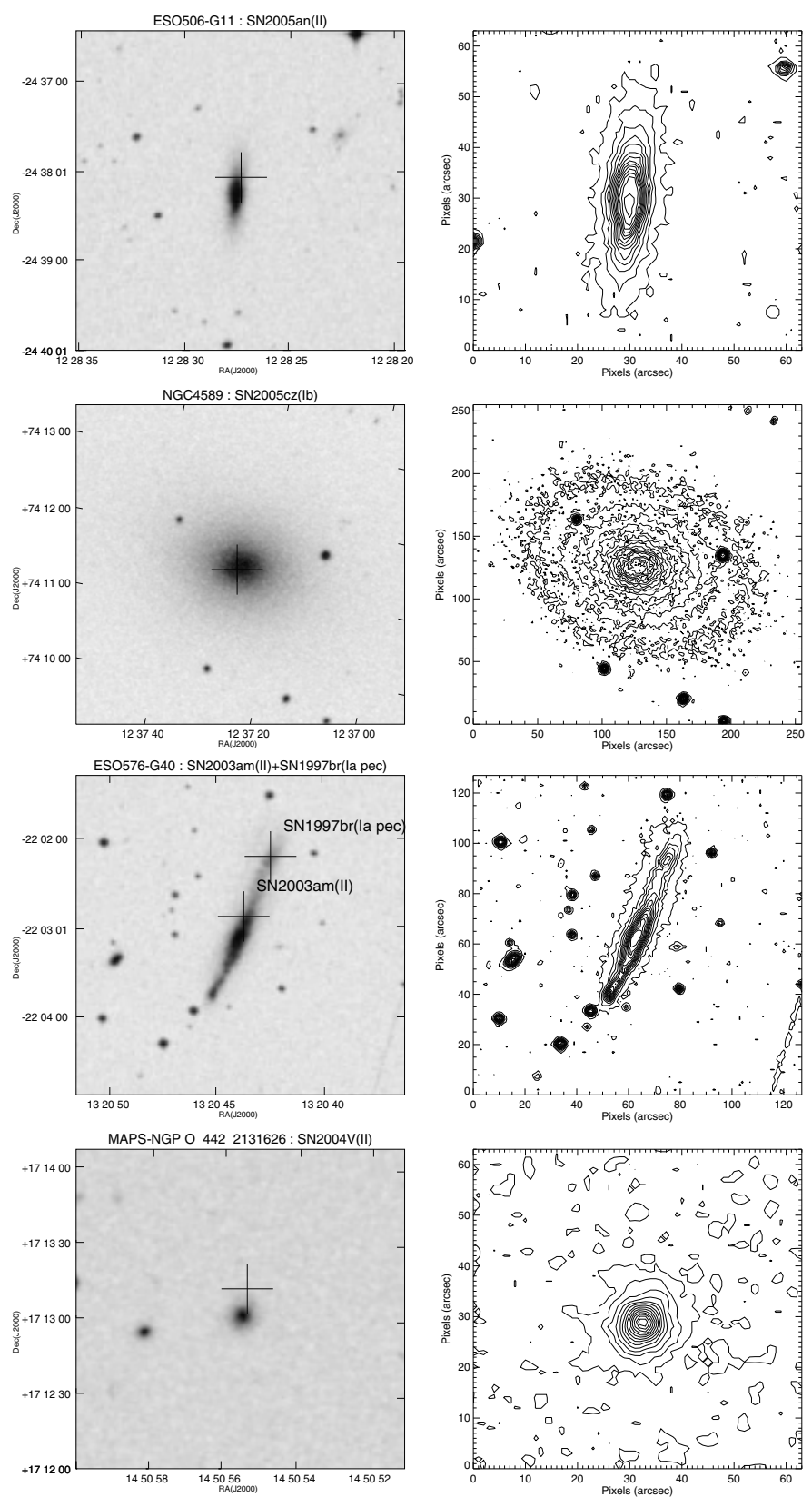

Fig. 1. e) continued.

According to Huchtmeier \& Richter (1989), the galaxy is a strong HI source. This object is included in the catalog of flat disk-like galaxies by Karachentsev et al. (1993) under the name 2MFGC5057. Our imaging study confirms the presence of latetype spiral structure but since this object is highly inclined $(r / R=0.42)$ its morphological classification is uncertain and we prefer a Sc classification for it. Two SNe have been discovered in this galaxy, the type Ia SN1996bv, and the type Ic: (revised classification, Filippenko et al. 2003) SN2003kb. Both SNe are projected on the disk of the galaxy. The core collapse SN2003kb is located on a spiral arm.

NGC 2274. The ASC and NED agree assigning type E to this galaxy. According to Karachentseva (1973) this object, together with NGC 2275, is a member of an isolated pair of galaxies (KPG 118). Huchtmeier \& Richter (1989) included it in their catalogue of HI sources. Cutri \& McAlary (1985) reported upper limits of $10 \mu$ emission from the nuclei of both galaxies, which is indicative of non-stellar radiation. Sign of interaction with its
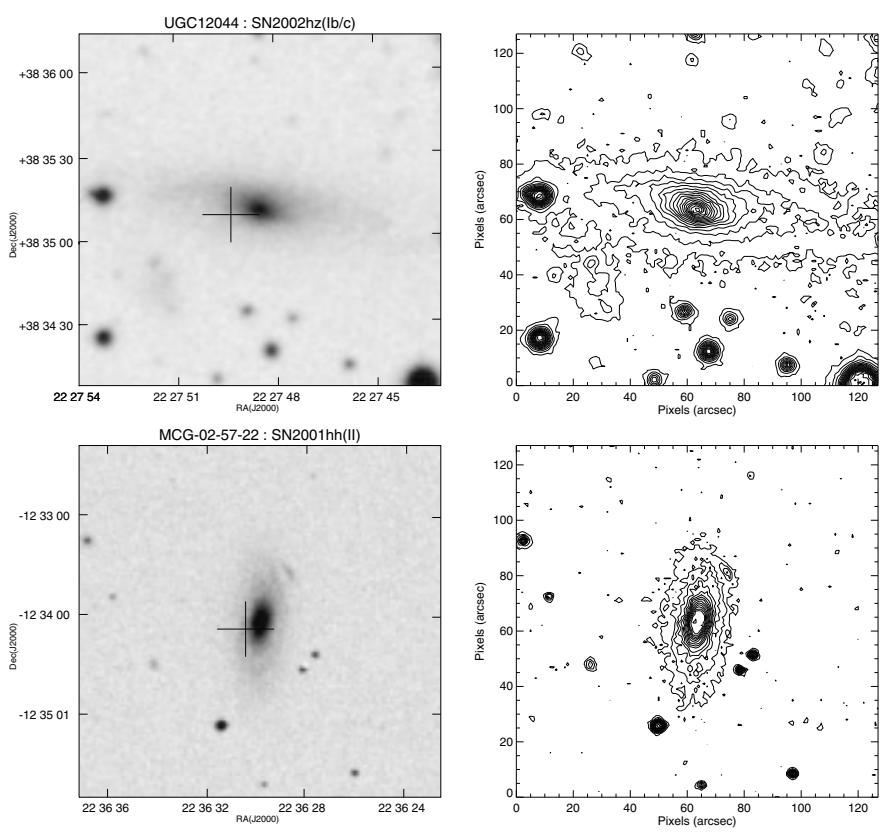

Fig. 1. f) continued.

northern neighbor is visible only in the outer envelope but no sign of a tidal effect is evident. Our analysis confirms its E morphological type. The position of SN2005md is close to the broad low-surface-brightness bridge connecting both galaxies in the system. The early SN classification as type II was based on the presence of a blue featureless continuum (Modjaz et al. 2005a). About 2 years later a new SN within $0.5^{\prime \prime}$ from SN2005md or a rebrightening of $2005 \mathrm{md}$ itself has been detected (Li et al. 2008). Unfortunately no spectral information is available to prove the nature of this object. Therefore the possibility that the two events are related super-outbursts of a LBV is not ruled out and has been proposed in the case of SN2006jc (Foley et al. 2007; Pastorello et al. 2007).

NGC 2768. According to ASC this is an E6. van den Bergh et al. (2003) assign type E3/Sa, indicating doubts about its type. A dual E-Sa class was also assigned by Maund \& Smartt (2005). In NED, its morphology is reported as S0_1/2 and its activity class is LINER. The NED information is from The Carnegie Atlas of Galaxies (Sandage \& Bedke 1994) in which the following description is presented: "The definite outer envelope of NGC 2768 surrounding an E6 bulge makes S0 classification certain. Very subtle dust patches on either side of the major axis at the ends of the minor axis requires $S 0 \_1 / 2$ classification". This galaxy has blue integral colours $(U-B=-0.43$ and $B-V=0.92$ ) and it is registered as a CO (Wiklind et al. 1995) and HI emission source (Huchtmeier et al. 1995) and a source of $1.4 \mathrm{GHz}$ radio continuum emission (Condon et al. 1998). Perhaps the most interesting information relates to the nuclear and near-nuclear regions of this galaxy: according to Michard \& Marchal (1994) "... the inner isophotes of the galaxy are boxy, but become disky in the range of 75-115 arcsecs". According to Lauer et al. (2005) and McDermid et al. (2006) this galaxy has a dust-obscured central region (spiral dust lanes), central disky gas distribution and a LINER nucleus with strong emission lines. The stellar population in the central region of this galaxy is relatively young. The resolution of our images was not sufficient to analyze in detail the central nuclear structure, but from its outer structure this object is a classical S0 type. SN2000ds, classified as type Ib (Filippenko \& Chornock 2000) is located far from the peculiar nuclear region of the galaxy. Detailed study of the SN 
position by van Dyk et al. (2003) shows that “... reddish clusters of stars may be evident near the position of the $\mathrm{SN}$ at the 3-4 $\sigma$ level".

IC 2461. van den Bergh et al. (2003) classified this galaxy as $\mathrm{S} 0$, but noted that it is viewed edge-on and so the classification is somewhat uncertain. ASC classified it as Sb, and NED assigned Sbc. This galaxy is included in the catalog of flat disk-like galaxies (Karachentsev et al. 1993) under the name of 2MFGC7243 and, according to Goad \& Roberts (1981), has a well-defined regular velocity field typical for late-type spiral galaxies. According to images and spectra from the SDSS DR6 (SDSS J091958.02+371128.5) it is an edge-on Sbc galaxy with welldelineated dust lanes and with spectra rich in strong emission lines ( $\mathrm{H} \alpha$, [OIII], etc.). This object is a strong source of HI emission (Theureau et al. 2007) and $1.4 \mathrm{GHz}$ radio continuum emissions (Condon et al. 1998). Our imaging study confirms the Sbc morphological classification. SN2002bx is a type II SN discovered soon after maximum (Matheson et al. 2002) and positioned in the far edge of the disk.

VIIIZw140. No galaxy type is reported in the ASC for this galaxy which was classified as E3 by van den Bergh et al. (2005). NED assigned type E. According to Paturel et al. (2003), it is an $\mathrm{HI}$ galaxy and also has $1.4 \mathrm{GHz}$ continuum radio emission (Condon et al. 1998). In our images, the central region of the galaxy are over-exposed whereas in the outer regions the surface brightness declines from a regular distribution and forms elongations to the north and south and the existence of two embryonic spiral arms is suspected. SN2004X, classified as a SN II (Filippenko et al. 2004), is positioned in the outer envelope of the galaxy. No specific morphological structure is present at the $\mathrm{SN}$ position.

NGC 3720. van den Bergh et al. (2003) classified this galaxy as E1, but noted that its type might be $\mathrm{Sa}$, which is the ASC classification. This galaxy is listed as SAa in NED, and Karachentsev (1973) identified it as a member of an isolated pair (KPG289B). According to Sandage \& Bedke (1994) "The nucleus of this galaxy is small and bright. The multiple arms are smooth at the resolution of the present plate material. The galaxy is clearly a spiral". Shi et al. (2006) described it as SAa with emission line spectra. The blue integral colours $(U-B=-0.04$ and $B-V=0.76$ ) of the galaxy are typical for Sb spirals (e.g., Huchra 1977). According to Paturel et al. (2003) this galaxy has detectable HI emission and according to Condon et al. (1998) is $1.4 \mathrm{GHz}$ continuum radio emission source. This object has been observed in the SDSS (SDSS J113221.83+004816.5) and it is obviously an Sa-type spiral galaxy. In our images the nuclear regions are over-exposed, but the structure of the circumnuclear and outer regions confirms its spiral nature. We suggest a Sa classification. SN2002at, classified as possible young SN II (Filippenko \& Barth 2002), appeared on an outer narrow spiral arm of the galaxy.

IC 3203. van den Bergh et al. (2003) classified this galaxy as S0: but noted, as for IC 2461, that it is viewed edge-on and therefore has a somewhat uncertain classification. In both the ASC and NED it is reported as Sb. In the Gold Mine Database (e.g., Gavazzi et al. 2006) it is described as an Sb galaxy with emission line spectra. This galaxy is included in the catalog of 2MASSselected flat disk-like galaxies (Mitronova et al. 2004) under the name of 2MFGC9726. According to Paturel et al. (2003) it is an HI object. Our imaging study confirms the Sb morphological type for this galaxy. SN2003ac, confirmed to be a rare SN IIb (Filippenko \& Chornock 2003b), is located in the outer parts of the disk.
ESO506-G011. ASC and NED both report this as an S0 galaxy. da Costa et al. (1998) agree, assigning it (-2) S0 type. The analysis of this edge-on object shows the presence of a prominent bulge and disk structure. We suggest to classify it as an early (possibly Sb type) spiral galaxy. SN2005an was an early SN II (Modjaz et al. 2005b) located in the disk of this galaxy.

NGC 4589. According to the ASC and NED this is an E2 galaxy. Our images do show a surface brightness distribution resembling an elliptical galaxy. According to Theureau et al. (2007) it is a strong $21 \mathrm{~cm}$ HI source. In a study of the gas and stellar kinematics of this galaxy, Ravindranath et al. (2001) concluded that it is a merger remnant (see also Moellenhoff \& Bender 1989) with LINER-type nuclear activity (Larkin et al. 1998). Studies by Michard \& Marchal (1994), Carollo et al. (1997) and Lauer et al. (2005) show the existence of large, chaotic, and disorganized opaque dust patches (in and outside of 4 " fields), forming asymmetric patterns, which might indicate a disk. Summarizing above data we conclude that this galaxy is a merger remnant with an outer structure similar to an elliptical galaxy. The object is a source of $1.4 \mathrm{GHz}$ continuum radio emission (Condon et al. 1998). SN2005cz is a type Ib SN (Leonard 2005) $17^{\prime \prime}$ far from the nucleus. The peculiarities in the central regions of this galaxy (and reaching out to the location of the $\mathrm{SN}$ ) are possibly associated with star formation (e.g., Kauffmann et al. 2003).

ESO576-G040. According to van den Bergh et al. (2003) this is an SOt type galaxy. NED assigned type SBd? pec. According to Corwin, de Vaucouleurs, \& de Vaucouleurs (1985) "Galaxy has over-exposed bar? Pretty smooth north arm, very knotty south arm". Gallagher et al. (2005) classified this object as an SBd type spiral with emission line spectra. Following Meyer et al. (2004) it is HIPASS HI source J1320-22. Our imaging of this highly-inclined galaxy confirms its spiral nature. A bar and two spiral arm structures can be identified therefore we prefer to classify this galaxy as SBc type. In this galaxy two SNe have been discovered: the type II SN2003am (Filippenko \& Chornock 2003b) located on a bright condensation of a northern spiral arm and the peculiar type Ia SN1997br (Li et al. 1999) in the disk of the galaxy to the north of the nucleus and close to another bright HII region.

MAPS-NGP O_442_2131626. van den Bergh et al. (2005) classified this galaxy as E0, the same morphological type (E:0) assigned by NED. In the literature there are no published data. Our morphological study reveals a low-contrast spiral arm structure pointing in a north/north-west direction. We classify the galaxy as Sa type. The type II SN2004V (Filippenko et al. 2004) is positioned close to the faint spiral arm structure.

UGC 12044. van den Bergh et al. (2003) classified this galaxy as Sb/S0. The ASC, NED, and Huchra et al. (1999) all agree that this is an Sab galaxy. According to Paturel et al. (2003) $\mathrm{HI}$ has been detected in it. Our imaging study confirms the Sab morphological classification. The type Ib SN2002hz (Filippenko \& Chornock 2003a) is projected on the disk but not associated with particular structures.

MCG-02-57-22. In the ASC this galaxy is classified as S0, whereas according to van den Bergh et al. (2005) it is an Sa. $\mathrm{NED}$ assigned an $\left(\mathrm{R}^{\prime}\right) \mathrm{SB}^{\wedge} 0^{\wedge}$ pec? morphological type while according to Karachentseva \& Karachentsev (2000) it is an $\mathrm{Sa}$, and a member of an isolated triplet (KTS70A). da Costa et al. (1998) classified it as Im (10) galaxy. Following to Fricke \& Kollatschny (1989) it is a faint $\mathrm{H} \alpha$ emitter. Our morphological study confirms the spiral nature of this galaxy (Sa classification). 
The Las Campanas $6.5 \mathrm{~m}$ telescope image ${ }^{4}$ of SN2001hh, a normal type II SN (Matheson et al. 2001), shows it on the inner edge of a southern spiral arm, thus supporting our claim.

\section{Discussion and conclusions}

It is generally believed (e.g., van den Bergh \& Tammann 1991; Cappellaro et al. 1999) that the hosts of core collapse SNe are objects with young stellar population (generally spiral or irregular galaxies) while the old stellar population of early type galaxies can produce only $\mathrm{SNe}$ Ia. Nevertheless among the morphologically-classified host galaxies of CCSNe, we have noted 22 cases in which the host has been classified as elliptical or S0, in apparent contradiction to this conventional view. Indeed it is well known that the morphological classification of galaxies has margins of subjectivity. For instance van den Bergh et al. (2003) have noted that "...late type galaxies of unusually high surface brightness may be misclassified as being of early type", and mention several additional minor issues with the classifications.

For the 22 cases in which the host galaxies of CCSNe have been reported as early types we have assembled imaging and searched the literature for additional information. We found that 14 hosts clearly ought to be re-classified as spirals, for three galaxies the presence of spiral structure is suspected, one galaxy has been classified as irregular, one as a barred galaxy with an outer ring structure. We share the view with van den Bergh et al. (2003) that high surface brightness in galaxies plays a crucial role in misclassification, particularly for largely inclined galaxies. There are no detailed studies on the surface brightness of our sample galaxies but we outline that six out of 22 galaxies are largely inclined objects. Since radial velocity may be another important factor influencing morphological classification we have compared the mean radial velocities of the total sample of CCSNe hosts in ASC with available recession velocity and morphology (910 galaxies) with those of the sample of 22 galaxies. The mean radial velocity of the total sample is $4935 \pm 3153 \mathrm{~km} \mathrm{~s}^{-1}$ to be compared with $4996 \pm 2434 \mathrm{~km} \mathrm{~s}^{-1}$ of our galaxies. This means that the uncertain classification was not due to the distance effect.

Other parameters, in addition to the morphological type, bar and ring are indicators of young stellar populations. In the sample there is one merger (NGC 4589) with elliptical outer isophotes and active nuclear region, which may also have enhanced star formation (e.g., Kauffmann et al. 2003). In fact, the $\mathrm{SN}$ in this galaxy is close to the nucleus. Another galaxy (NGC 2768) has been confirmed as S0 with a central region showing dust and a disky central gas distribution. This galaxy has a powerful LINER nucleus, and may also be the remnant of a merger. The existence of strong $\mathrm{HI}$ and $\mathrm{CO}$ emission, and the presence of dust and ionized gas supports the presence of a young stellar population. The core collapse $\mathrm{SN}$ in this galaxy is possibly associated with the red star clusters present. Only one galaxy of the 22, NGC 2274, remains confirmed with an elliptical morphology without any inner structural peculiarity. This galaxy is a member of an isolated pair, and appears to be in interaction with a close neighbor spiral. NGC 2274 may possibly have accreted some gas during a tidal encounter with this gasrich neighbor, thereby accounting for the presence of massive stars. Despite the low number statistics it is interesting to note the unusually high ratio SN Ibc / SN II = 2/1 found in early

\footnotetext{
4 http://cfa-www .harvard. edu/supernova/images/2001hh. jpg
}

type galaxies with respect to other galaxy types (e.g., Table 5 of Barbon et al. 1999).

Our sample of 22 galaxies includes $17 \mathrm{HI}$ sources (more than $77 \%$ of the total sample). It has been shown many times that the presence of $\mathrm{HI}$ emission is correlated with star formation, and the HI is generally found in close proximity to star-forming regions (e.g., Helmboldt 2007). A high HI detection rate among our sample galaxies is consistent with the presence of a young stellar population in these objects. Our sample includes also 8 $1.4 \mathrm{GHz}$ continuum radio sources. According to the criterion adopted by Della Valle et al. (2005), among 8 radio sources detected 2 (NGC 838, UGC 2836) are radio-loud and 6 (IC 1429, NGC 2768, NGC 3720, IC 2461, VIIIZw140 and NGC 4589) are radio-faint. In two cases (NGC 2768, NGC 4589) the LINER AGNs might contribute to the radio emission (e.g., Falcke et al. 2000). In the 6 remaining cases the radio emission might witness recent star formation (e.g., Rosa-González et al. 2007). It is interesting to note that two galaxies in the sample are UV excess Markarian galaxies, and three have LINER type AGNs. In one case (NGC 1260 with SN2006gy), the SN is located in the inner core of the galaxy characterized by extended $\mathrm{H} \alpha$ emission, consistent with the supposed large mass of the progenitor.

In summary:

1. In 17 cases, the "early type" host is a misclassified spiral galaxy.

2. In one case the host galaxy is a misclassified irregular, and in another it is a misclassified ring galaxy.

3. Three galaxies retain the early type classification: NGC 4579 a merger remnant with elliptical outer isophotes; NGC 2768, a S0 with LINER nucleus, possibly the remnant of a merger; NGC 2274 an elliptical with no morphological peculiarity which anyway appears to be in close interaction with a neighbour.

In conclusion, the presence of CCSNe in early type galaxies can be interpreted as an additional indication that residual star formation episodes take place also in E and S0 galaxies due to merging/accretion or interaction with close neighbours. In other words the morphology of a galaxy is the most direct and immediate indicator of its general stellar content and star formation history. Nevertheless the morphology is inadequate to describe local SF episodes occurring at small scale.

The available small statistics is inadequate to perform a detailed analysis of the frequency of CCSNe in early type galaxies, hence to test whether the presence of CCSNe is compatible with the existence of a "prompt" population of SN Ia as invoked by Mannucci et al. (2006).

Acknowledgements. A.H. acknowledges the hospitality of the Institut d'Astrophysique de Paris (France). The work of A.A. Hakobyan at IAP was supported by PICS France-Armenie and the grant of French Government. A.P. acknowledgments the hospitality of the Space Telescope Science Institute during his stay as visiting scientist supported by the Director's Discretionary Research Fund. A.P. wishes to thank the Institut d'Astrophysique de Paris (France) hospitality and support (via the EARA network) during the last stage of this work. M.T. acknowledges the support of the Italian Ministry of University and Research via PRIN RIN 2006 n.2006022731 002. Funding for the SDSS and SDSS-II has been provided by the Alfred P. Sloan Foundation, the Participating Institutions are the National Science Foundation, the US Department of Energy, the National Aeronautics and Space Administration, the Japanese Monbukagakusho, the Max Planck Society, and the Higher Education Funding Council for England. The SDSS Web Site is http:// www.sdss.org/. This research has made use of NASA/IPAC Extragalactic Database (NED), which is operated by the Jet Propulsion Laboratory, California Institute of Technology, under contract with the National Aeronautics and Space Administration, and HyperLeda (Leon-Meudon Extragalactic Database, http://cismbdm.univ-lyon1.fr/ hyperleda/). The Digitized Sky 
Survey was produced at the Space Telescope Science Institute under US Government grant NAG W-2166. The images of this survey are based on photographic data obtained using the Oschin Schmidt Telescope on Palomar Mountain and the UK Schmidt Telescope. The plates were processed into the present digital form with the permission of these institutions. Finally, we are especially grateful to our referee for promptly and carefully reading the manuscript and making several constructive comments which have improved the paper quite significantly.

\section{References}

Adelman-McCarthy, J. K., Agüeros, M. A., Allam, S. S., et al. 2007, ApJS, 172, 634

Andreon, S., Davoust, E., \& Poulain, P. 1997, A\&AS, 126, 67

Barbon, R., Buondí, V., Cappellaro, E., \& Turatto, M. 1999, A\&AS, 139, 531

Bartunov, O. S., Tsvetkov, D. Y., \& Filimonova, I. V. 1994, PASP, 106, 1276

Caldwell, N., Rose, J. A., \& Concannon, K. D. 2003, AJ, 125, 2891

Cappellaro, E., Evans, R., \& Turatto, M. 1999, A\&A, 351, 459

Carollo, C. M., Franx, M., Illingworth, G. D., \& Forbes, D. A. 1997, ApJ, 481, 710

Condon, J. J., Cotton, W. D., Greisen, E. W., et al. 1998, AJ, 115, 1693

Corwin, H. G., de Vaucouleurs, A., \& de Vaucouleurs, G. 1985, Southern galaxy catalogue. A catalogue of 5481 galaxies south of declination -17 grad. found on $1.2 \mathrm{~m}$ UK Schmidt IIIa J plates (University of Texas Monographs in Astronomy, Austin: University of Texas)

Coziol, R., Demers, S., Pena, M., \& Barneoud, R. 1994, AJ, 108, 405

Cutri, R. M., \& McAlary, C. W. 1985, ApJ, 296, 90

da Costa, L. N., Willmer, C. N. A., Pellegrini, P. S., et al. 1998, AJ, 116, 1

de Carvalho, R. R., \& Coziol, R. 1999, AJ, 117, 1657

de Vaucouleurs, G., de Vaucouleurs, A., Corwin, Jr., H. G., et al. 1991, Third Reference Catalogue of Bright Galaxies, Vol. 1-3, XII, (Berlin Heidelberg New York: Springer-Verlag)

Della Valle, M., Panagia, N., Padovani, P., et al. 2005, ApJ, 629, 750

Falcke, H., Nagar, N. M., Wilson, A. S., \& Ulvestad, J. S. 2000, ApJ, 542, 197

Filippenko, A. V., \& Barth, A. J. 2002, IAU Circ., 7829, 3

Filippenko, A. V., \& Chornock, R. 2000, IAU Circ., 7511, 2

Filippenko, A. V., \& Chornock, R. 2001, IAU Circ., 7571, 2

Filippenko, A. V., \& Chornock, R. 2003a, IAU Circ., 8051, 2

Filippenko, A. V., \& Chornock, R. 2003b, IAU Circ., 8085, 2

Filippenko, A. V., \& Chornock, R. 2003c, IAU Circ., 8246, 4

Filippenko, A. V., Li, W. D., Treffers, R. R., \& Modjaz, M. 2001, in Small Telescope Astronomy on Global Scales, ed. B. Paczynski, W.-P. Chen, \& C. Lemme, ASP Conf. Ser., 246, IAU Colloq. 183, 121

Filippenko, A. V., Foley, R. J., \& Swift, B. J. 2003, IAU Circ., 8243, 2

Filippenko, A. V., Chornock, R., \& Foley, R. J. 2004, IAU Circ., 8287, 2

Foley, R. J., \& Filippenko, A. V. 2002, IAU Circ., 8031, 4

Foley, R. J., Smith, N., Ganeshalingam, M., et al. 2007, ApJ, 657, L105

Fricke, K. J., \& Kollatschny, W. 1989, A\&AS, 77, 75

Gallagher, J. S., Garnavich, P. M., Berlind, P., et al. 2005, ApJ, 634, 210

Gavazzi, G., Boselli, A., Cortese, L., et al. 2006, A\&A, 446, 839

Goad, J. W., \& Roberts, M. S. 1981, ApJ, 250, 79

Green, D. W. E. 2006, Central Bureau Electronic Telegrams, 584, 2

Greggio, L., Renzini, A., \& Daddi, E. 2008, ArXiv e-prints, 805

Hamuy, M. 2001, Ph.D. Thesis, Univ. Arizona

Hamuy, M. 2003, ApJ, 582, 905

Hau, G. K. T., Ferguson, H. C., Lahav, O., \& Lynden-Bell, D. 1995, MNRAS, 277,125

Heger, A., Woosley, S. E., Fryer, C. L., \& Langer, N. 2003, in From Twilight to Highlight: The Physics of Supernovae, ed. W. Hillebrandt, \& B. Leibundgut, 3

Helmboldt, J. F. 2007, MNRAS, 379, 1227

Hendry, M. A., Smartt, S. J., Crockett, R. M., et al. 2006, MNRAS, 369, 1303

Hickson, P., Kindl, E., \& Auman, J. R. 1989, ApJS, 70, 687

Huchra, J. P. 1977, ApJS, 35, 171

Huchra, J., Latham, D. W., da Costa, L. N., Pellegrini, P. S., \& Willmer, C. N. A. 1993, AJ, 105, 1637

Huchra, J. P., Vogeley, M. S., \& Geller, M. J. 1999, ApJS, 121, 287

Huchtmeier, W. K., \& Richter, O.-G. 1989, A General Catalog of HI Observations of Galaxies. The Reference Catalog., 350 (Berlin Heidelberg: Springer-Verlag)

Huchtmeier, W. K., Sage, L. J., \& Henkel, C. 1995, A\&A, 300, 675

Jha, S., Garnavich, P., Challis, P., Kirshner, R., \& Berlind, P. 1999, IAU Circ., 7280,2

Kandalyan, R. A. 2003, A\&A, 398, 493

Karachentsev, I. D. 1973, Astrofizicheskie Issledovaniia Izvestiya Spetsial'noj Astrofizicheskoj Observatorii, 5, 3
Karachentsev, I. D., Karachentseva, V. E., \& Parnovskij, S. L. 1993, Astronomische Nachrichten, 314, 97

Karachentseva, V. E. 1973, Soobshcheniya Spetsial'noj Astrofizicheskoj Observatorii, 8, 3

Karachentseva, V. E., \& Karachentsev, I. D. 2000, Astronomy Reports, 44, 501 Kauffmann, G., Heckman, T. M., Tremonti, C., et al. 2003, MNRAS, 346, 1055 Kennicutt, Jr., R. C. 1989, ApJ, 344, 685

Larkin, J. E., Armus, L., Knop, R. A., Soifer, B. T., \& Matthews, K. 1998, ApJS, 114,59

Lauer, T. R., Faber, S. M., Gebhardt, K., et al. 2005, AJ, 129, 2138

Leonard, D. C. 2005, IAU Circ., 8579, 2

Li, W., Filippenko, A. V., Mostardi, R., et al. 2008, Central Bureau Electronic Telegrams, 1265, 1

Li, W. D., Qiu, Y. L., Qiao, Q. Y., et al. 1999, AJ, 117, 2709

Mannucci, F., Della Valle, M., \& Panagia, N. 2006, MNRAS, 370, 773

Matheson, T., Jha, S., Kirshner, R., \& Falco, E. 2001, IAU Circ., 7770, 4

Matheson, T., Jha, S., Challis, P., Kirshner, R., \& Calkins, M. 2002, IAU Circ., 7868, 2

Mauch, T., \& Sadler, E. M. 2007, MNRAS, 375, 931

Maund, J. R., \& Smartt, S. J. 2005, MNRAS, 360, 288

Maza, J., \& van den Bergh, S. 1976, ApJ, 204, 519

McDermid, R. M., Emsellem, E., Shapiro, K. L., et al. 2006, MNRAS, 373, 906

Meusinger, H., Brunzendorf, J., \& Krieg, R. 2000, A\&A, 363, 933

Meyer, M. J., Zwaan, M. A., Webster, R. L., et al. 2004, MNRAS, 350, 1195

Michard, R. \& Marchal, J. 1994, A\&AS, 105, 481

Mitronova, S. N., Karachentsev, I. D., Karachentseva, V. E., Jarrett, T. H., \& Kudrya, Y. N. 2004, Bull. Special Astrophys. Obs., 57, 5

Modjaz, M., Kirshner, R., Challis, P., Blondin, S., \& Berlind, P. 2005a, IAU Circ., 8650, 2

Modjaz, M., Kirshner, R., Challis, P., \& Hao, H. 2005b, IAU Circ., 8491, 2

Moellenhoff, C., \& Bender, R. 1989, A\&A, 214, 61

Naim, A., Lahav, O., Buta, R. J., et al. 1995, MNRAS, 274, 1107

Navasardyan, H., Petrosian, A. R., Turatto, M., Cappellaro, E., \& Boulesteix, J. 2001, MNRAS, 328, 1181

Noordermeer, E., van der Hulst, J. M., Sancisi, R., Swaters, R. A., \& van Albada, T. S. 2005, A\&A, 442, 137

Ofek, E. O., Cameron, P. B., Kasliwal, M. M., et al. 2007, ApJ, 659, L13

Pastorello, A., Taubenberger, S., Patat, F., et al. 2005, IAU Circ., 8467, 2

Pastorello, A., Smartt, S. J., Mattila, S., et al. 2007, Nature, 447, 829

Paturel, G., Theureau, G., Bottinelli, L., et al. 2003, A\&A, 412, 57

Petrosian, A., Navasardyan, H., Cappellaro, E., et al. 2005, AJ, 129, 1369

Petrosian, A., McLean, B., Allen, R. J., \& MacKenty, J. W. 2007, ApJS, 170, 33

Petrosian, A., McLean, B., Allen, R., Kunth, D., \& Leitherer, C. 2008, ApJS, 175,86

Puckett, T., Peoples, M., Joubert, N., et al. 2006, IAU Circ., 8741, 1

Ravindranath, S., Ho, L. C., Peng, C. Y., Filippenko, A. V., \& Sargent, W. L. W. 2001, AJ, 122, 653

Rosa-González, D., Schmitt, H. R., Terlevich, E., \& Terlevich, R. 2007, ApJ, 654,226

Ryan-Weber, E. V., Meurer, G. R., Freeman, K. C., et al. 2004, AJ, 127, 1431

Sandage, A., \& Bedke, J. 1994, The Carnegie atlas of galaxies (Washington, DC: Carnegie Institution of Washington with The Flintridge Foundation)

Shi, L., Gu, Q. S., \& Peng, Z. X. 2006, A\&A, 450, 15

Smith, N., Li, W., Foley, R. J., et al. 2007, ApJ, 666, 1116

Sulentic, J. W., Verdes-Montenegro, L., Bergond, G., et al. 2006, A\&A, 449, 937

Taubenberger, S., Pastorello, A., Mazzali, P. A., et al. 2006, MNRAS, 371, 1459

Theureau, G., Hanski, M. O., Coudreau, N., Hallet, N., \& Martin, J.-M. 2007, A\&A, 465, 71

Turatto, M., Benetti, S., \& Pastorello, A. 2007, in Amer. Inst. Phys. Conf. Ser., ed. S. Immler, \& R. McCray, Amer. Inst. Phys. Conf. Ser., 937, 187

van den Bergh, S., \& Tammann, G. A. 1991, ARA\&A, 29, 363

van den Bergh, S., Li, W., \& Filippenko, A. V. 2002, PASP, 114, 820

van den Bergh, S., Li, W., \& Filippenko, A. V. 2003, PASP, 115, 1280

van den Bergh, S., Li, W., \& Filippenko, A. V. 2005, PASP, 117, 773

van Dyk, S. D. 1992, AJ, 103, 1788

van Dyk, S. D. 2005, in 1604-2004: Supernovae as Cosmological Lighthouses,

ed. M. Turatto, S. Benetti, L. Zampieri, \& W. Shea, ASP Conf. Ser., 342, 115

van Dyk, S. D., Peng, C. Y., Barth, A. J., \& Filippenko, A. V. 1999, AJ, 118, 2331

van Dyk, S. D., Li, W., \& Filippenko, A. V. 2003, PASP, 115, 1

Verdes-Montenegro, L., Yun, M. S., Williams, B. A., et al. 2001, A\&A, 377, 812

Wiklind, T., Combes, F., \& Henkel, C. 1995, A\&A, 297, 643

Wong, D. S., Silverman, J. M., Pooley, D., \& Filippenko, A. V. 2006, IAU Circ., 8677,3

Woosley, S. E., \& Weaver, T. A. 1995, ApJS, 101, 181

Woosley, S. E., Blinnikov, S., \& Heger, A. 2007, Nature, 450, 390 